\title{
DSP FOR IN-VEHICLE AND MOBILE SYSTEMS
}




\section{DSP FOR IN-VEHICLE AND MOBILE SYSTEMS}

Edited by

\section{Hüseyin Abut}

Department of Electrical and Computer Engineering

San Diego State University, San Diego, California, USA

<abut@akhisar.sdsu.edu>

\section{John H.L. Hansen}

Robust Speech Processing Group, Center for Spoken Language Research Dept. Speech, Language \& Hearing Sciences, Dept. Electrical Engineering University of Colorado, Boulder, Colorado, USA

$<$ John.Hansen@colorado.edu>

\section{Kazuya Takeda}

Department of Media Science

Nagoya University, Nagoya, Japan

$<$ takeda@is.nagoya-u.ac.jp> 
eBook ISBN: $\quad$ 0-387-22979-5

Print ISBN: $\quad$ 0-387-22978-7

C2005 Springer Science + Business Media, Inc.

Print @2005 Springer Science + Business Media, Inc.

Boston

All rights reserved

No part of this eBook may be reproduced or transmitted in any form or by any means, electronic, mechanical, recording, or otherwise, without written consent from the Publisher

Created in the United States of America

Visit Springer's eBookstore at:

http://ebooks.kluweronline.com and the Springer Global Website Online at: http://www.springeronline.com 


\section{$\underline{\text { Dedication }}$}

To Professor Fumitada Itakura

This book, "DSP for In-Vehicle and Mobile Systems", contains a collection of research papers authored by prominent specialists in the field. It is dedicated to Professor Fumitada Itakura of Nagoya University. It is offered as a tribute to his sustained leadership in Digital Signal Processing during a professional career that spans both industry and academe. In many cases, the work reported in this volume has directly built upon or been influenced by the innovative genius of Professor Itakura.

While this outstanding book is a major contribution to our scientific literature, it represents but a small chapter in the anthology of technical contributions made by Professor Itakura. His purview has been broad. But always at the center has been digital signal theory, computational techniques, and human communication. In his early work, as a research scientist at the NTT Corporation, Itakura brought new thinking to bit-rate compression of speech signals. In partnership with Dr. S. Saito, he galvanized the attendees of the 1968 International Congress on Acoustics in Tokyo with his presentation of the Maximum Likelihood Method applied to analysis-synthesis telephony. The presentation included demonstration of speech transmission at 5400 bits/sec with quality higher than heretofore achieved. His concept of an allpole recursive digital filter whose coefficients are constantly adapted to predict and match the short-time power spectrum of the speech signal caused many colleagues to hurry back to their labs and explore this new direction. From Itakura's stimulation flowed much new research that led to significant advances in linear prediction, the application of autocorrelation, and eventually useful links between cepstral coefficients and linear prediction. Itakura was active all along this route, contributing among other ideas, new knowledge about the Line Spectral Pair (LSP) as a robust means for encoding predictor coefficients. A valuable by-product of his notion of adaptively matching the power spectrum with an all-pole digital filter gave rise to the Itakura-Saito distance measure, later employed in speech recognition as well as a criterion for low-bit-rate coding, and also used extensively in evaluating speech enhancement algorithms.

Itakura's originality did not escape notice at Bell labs. After protracted legalities, a corporate arrangement was made for sustained exchange of research scientists between ATT and NTT. Fumitada Itakura was the first to initiate the program, which later encompassed such notables as Sadaoki Furui, Yoh ichi Tohkura, Steve Levenson, David Roe, and subsequent others from 
both organizations. At Bell Labs during 1974 and -75, Fumitada ventured into automatic speech recognition, implementing an airline reservation system on an early laboratory computer. Upon his return to his home company Dr. Itakura was given new responsibilities in research management, and his personal reputation attracted exceptional engineering talent to his vibrant organization.

Following fifteen years of service with NTT, the challenges of academe beckoned, and Dr. Itakura was appointed Professor of Electrical Engineering in Nagoya University - the university which originally awarded his $\mathrm{PhD}$ degree. Since this time he has led research and education in Electrical Engineering, and Acoustic Signal Processing, all the while building upon his expertise in communications and computing. Sophisticated microphone systems to combat noise and reverberation were logical research targets, as exemplified by his paper with colleagues presented in this volume. And, he has continued management responsibilities in contributing to the leadership of the Nagoya University Center for Integrated Acoustic Information Research (CIAIR).

Throughout his professional career Professor Itakura has steadily garnered major recognition and technical awards, both national and international. But perhaps none rivals the gratification brought by the recognition bestowed by his own country in 2003 -- when in formal ceremony at the Imperial Palace, with his wife Nobuko in attendance, Professor Itakura was awarded the coveted Shiju-hosko Prize, also known as the Purple Ribbon Medal.

To his stellar record of career-long achievement we now add the dedication of this modest technical volume. Its pages are few by comparison to his accomplishments, but the book amply reflects the enormous regard in which Professor Fumitada Itakura is held by his colleagues around the world.

\section{Jim Flanagan}

Rutgers University 


\section{Table of Contents}

Dedication $\quad$ V

List of Contributors $\quad$ xi

Preface xiii

Chapter $1 \quad 1$

Construction and Analysis of a Multi-layered In-car Spoken Dialogue Corpus

Nobuo Kawaguchi, Shigeki Matsubara, Itsuki Kishida, Yuki Irie,

Hiroya Murao, Yukiko Yamaguchi, Kazuya Takeda, Fumitada Itakura

Center for Integrated Acoustic Information Research,

Nagoya University, Japan

Chapter 2

CU-Move: Advanced In-Vehicle Speech Systems for

\section{Route Navigation}

John H.L. Hansen, Xianxian Zhang, Murat Akbacak, Umit H. Yapanel,

Bryan Pellom, Wayne Ward, Pongtep Angkititrakul

Robust Speech Processing Group, Center for Spoken Language

Research, University of Colorado, Boulder, Colorado, USA

Chapter 3

A Spoken Dialog Corpus for Car Telematics Services

Masahiko Tateishi ${ }^{1}$, Katsushi Asami ${ }^{1}$, Ichiro Akahori ${ }^{1}$, Scott Judy ${ }^{2}$,

Yasunari Obuchi $^{3}$, Teruko Mitamura ${ }^{2}$, Eric Nyberg ${ }^{2}$, and Nobuo Hataoka ${ }^{4}$

${ }^{I}$ Research Laboratories, DENSO CORPORATION, Japan

${ }^{2}$ Language Technologies Institute, Carnegie Mellon University, USA

${ }^{3}$ Advanced Research Laboratory, Hitachi Ltd., Japan

${ }^{4}$ Central Research Laboratory, Hitachi Ltd., Japan

Chapter 4

Experiences of Multi-speaker Dialogue System for Vehicular

Information Retrieval

Hsien-chang Wang ${ }^{1}$, Jhing-fa Wang ${ }^{2}$

${ }^{I}$ Department of Computer Science and Information Engineering,

Taiwan, R.O.C.

${ }^{2}$ Department of Electrical Engineering, Taiwan, R.O.C. 
Chapter 5

Robust Dialog Management Architecture using VoiceXML for

Car Telematics Systems

Yasunari Obuchi ${ }^{1}$, Eric Nyberg ${ }^{2}$, Teruko Mitamura ${ }^{2}$, Scott Judy ${ }^{2}$,

Michael Duggan ${ }^{3}$, Nobuo Hataoka ${ }^{4}$

${ }^{1}$ Advanced Research Laboratory, Hitachi Ltd., Japan,

${ }^{2}$ Language Technologies Institute, Carnegie Mellon University, USA

${ }^{3}$ Software Engineering Institute, Carnegie Mellon University, USA

${ }^{4}$ Central Research Laboratory, Hitachi Ltd., Japan

Chapter 6

Use of Multiple Speech Recognition Units in an In-car

Assistance System

Alessio Brutti ${ }^{1}$, Paolo Coletti ${ }^{1}$, Luca Cristoforetti ${ }^{1}$, Petra Geutner ${ }^{2}$,

Alessandro Giacomini ${ }^{1}$, Mirko Maistrello ${ }^{1}$, Marco Matassoni ${ }^{1}$,

Maurizio Omologo ${ }^{1}$, Frank Steffens ${ }^{2}$, Piergiorgio Svaizer ${ }^{1}$

${ }^{1}$ ITC-irst (Centro per la Ricerca Scientifica e Tecnologica), Italy

${ }^{2}$ Robert Bosch GmbH, Corporate Research and Development,

Germany

Chapter 7

Hi-speed Error Correcting Code LSI for Mobile phone

Yuuichi Hamasuna ${ }^{1}$, Masayasu Hata ${ }^{2}$, Ichi Takumi ${ }^{3}$

${ }^{l}$ DDS Inc., Japan; ${ }^{2}$ Chubu University, Japan;

${ }^{3}$ Nagoya Institute of Technology, Japan

Chapter 8

MCMAC as an Amplitude Spectral Estimator for Speech

Enhancement

Abdul Wahab ${ }^{1}$, Tan Eng Chong ${ }^{1}$, Hüseyin Abut $^{2}$

${ }^{I}$ School of Computer Engineering, Nanyang Technological

University, Singapore; ${ }^{2}$ ECE Department, San Diego State

University, USA

Chapter 9

Noise Robust Speech Recognition using Prosodic Information

Koji Iwano, Takahiro Seki, Sadaoki Furui

Department of Computer Science, Tokyo Institute of Technology,

Japan 
Chapter 10

Reduction of Diffuse Noise in Mobile and Vehicular Applications

Hamid Sheikhzadeh ${ }^{1}$, Hamid Reza Abutalebi ${ }^{2}$, Robert L. Brennan ${ }^{1}$, GeorgeH.Freeman ${ }^{3}$

${ }^{1}$ Dspfactory Ltd., Waterloo, Ontario, Canada;

${ }^{2}$ Electrical Engineering Dept., University of Yazd, Iran;

${ }^{3}$ Electrical and Computer Engineering, University of Waterloo,

Ontario, Canada

Chapter 11

169

Speech Enhancement based on F-Norm Constrained Truncated

SVD Algorithm

Guo Chen, Soo Ngee Koh, and Ing Yann Soon

School of Electrical \& Electronic Engineering, Nanyang

Technological University, Singapore

Chapter 12

Verbkey - A Single-Chip Speech Control for the Automobile Environment

Rico Petrick ${ }^{1}$, Diane Hirschfeld ${ }^{1}$, Thomas Richter ${ }^{1}$, Rüdiger Hoffmann ${ }^{2}$

${ }^{I}$ voice INTER connect GmbH,Dresden,Germany

${ }^{2}$ Laboratory of Acoustics and Speech Communication,

Dresden University of Technology

Chapter 13

Real-time Transmission of H.264 Video over 802.11B-based

Wireless ad hoc Networks

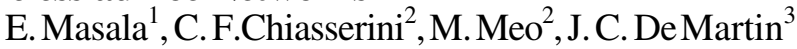

${ }^{I}$ Dipartimento di Automatica e Informatica, Politecnico di

Torino, Italy

${ }^{2}$ Dipartimento di Elettronica, Politecnico di Torino, Italy

${ }^{3}$ IEIIT-CNR, Politecnico di Torino, Italy

Chapter 14

DWT Image Compression for Mobile Communication

Lifeng Zhang, Tahaharu Kouda, Hiroshi Kondo, Teruo Shimomura Kyushu Institute of Technology, Japan 
Chapter 15

Link-adaptive Variable Bit-rate Speech Transmission over 802.11 Wireless LANs

Antonio Servetti ${ }^{1}$, Juan Carlos De Martin ${ }^{2}$

${ }^{I}$ Dipartimento di Automatica e Informatica, Politecnico di Torino, Italy

${ }^{2}$ IEIIT-CNR, Politecnico di Torino, Italy

Chapter 16

Joint Audio-Video Processing for Robust Biometric Speaker Identification in Car

Engin Erzin, Yücel Yemez, A. Murat Tekalp Multimedia, Vision and Graphics Laboratory, College of Engineering, Koç University, Turkey

Chapter 17

Is Our Driving Behavior Unique?

Kei Igarashi ${ }^{1}$, Kazuya Takeda ${ }^{2}$ and Fumitada Itakura ${ }^{1}$, Hüseyin Abut ${ }^{1,2}$ ${ }^{I}$ Centerfor Integrated Acoustic Information Research (CIAIR), Nagoya University, Japan

${ }_{2}^{2}$ ECE Department, San Diego State University, San Diego,CA USA

Chapter 18

Robust ASR Inside A Vehicle Using Blind Probabilistic Based Under-determined Convolutive Mixture Separation Technique

Shubha Kadambe HRLLaboratories, LLC, Malibu, CA, USA

Chapter 19

In-car Speech Recognition using Distributed Microphones

Tetsuya Shinde, Kazuya Takeda, Fumitada Itakura,

Graduate School of Engineering, Nagoya University, Japan

Index 


\section{List of Contributors}

Hüseyin Abut, San Diego State University, USA

Hamid R. Abutalebi, University of Yazd, Iran

Ichiro Akahori, Denso Corp., Japan

Murat Akbacak, University of Colorado at Boulder, USA

Pongtep Angkititrakul, University of Colorado at Boulder, USA

Katsushi Asami, Denso Corp., Japan

Robert L. Brennan, Dspfactory, Canada

Alessio Brutti, ITC-irst, Italy

Guo Chen, Nanyang Technological University, Singapore

Chiasserini F. Chiasserini, Politecnico di Torino, Italy

Tan Eng Chong, Nanyang Technological University, Singapore

Paolo Coletti, ITC-irst, Italy

Luca Cristoforetti, ITC-irst, Italy

Juan Carlos De Martin, Politecnico di Torino, Italy

Michael Duggan, Carnegie Mellon University, USA

Engin Erzin, Koç University, Turkey

George H. Freeman, University of Waterloo, Canada

Sadaoki Furui, Tokyo Institute of Technology, Japan

Petra Geutner, Robert Bosch, Germany

Alessandro Giacomini, ITC-irst, Italy

Yuuichi Hamasuna, DDS Inc., Japan

John H.L. Hansen, University of Colorado at Boulder, USA

Masayasu Hata, Chubu University, Japan

Nobuo Hataoka, Hitachi Ltd., Japan

Diane Hirschfeld, voice INTER connect, Germany

Rüdiger Hoffmann, Dresden University of Technology, Germany

Kei Igarashi, Nagoya University, Japan

Yuki Irie, Nagoya University, Japan

Fumitada Itakura, Nagoya University, Japan

Koji Iwano, Tokyo Institute of Technology, Japan

Scott Judy, Carnegie Mellon University, USA

Shubha Kadambe, HRL Laboratories, USA

Nobuo Kawaguchi, Nagoya University, Japan

Itsuki Kishida, Nagoya University, Japan

Soo Ngee Koh, Nanyang Technological University, Singapore 


\section{List of Contributors (cont.)}

Hiroshi Kondo, Kyushu Institute of Technology, Japan

Tahaharu Kouda, Kyushu Institute of Technology, Japan

Mirko Maistrello, ITC-irst, Italy

Enrico Masala, Politecnico di Torino, Italy

Marco Matassoni, ITC-irst, Italy

Shigeki Matsubara, Nagoya University, Japan

Michela Meo, Politecnico di Torino, Italy

Teruko Mitamura, Carnegie Mellon University, USA

Hiroya Murao, Nagoya University, Japan

Eric Nyberg, Carnegie Mellon University, USA

Yasunari Obuchi, Hitachi Ltd., Japan

Maurizio Omologo, ITC-irst, Italy

Bryan Pellom, University of Colorado at Boulder, USA

Rico Petrick, voice INTER connect, Germany

Thomas Richter, voice INTER connect, Germany

Takahiro Seki, Tokyo Institute of Technology, Japan

Antonio Servetti, Politecnico di Torino, Italy

Hamid Sheikhzadeh, Dspfactory, Canada

Teruo Shimomura, Kyushu Institute of Technology, Japan

Tetsuya Shinde, Nagoya University, Japan

Ing Yann Soon, Nanyang Technological University, Singapore

Frank Steffens, Robert Bosch, Germany

Piergiorgio Svaizer, ITC-irst, Italy

Kazuya Takeda, Nagoya University, Japan

Ichi Takumi, Nagoya Institute of Technology, Japan

Masahiko Tateishi, Denso Corp., Japan

A. Murat Tekalp, Koç University, Turkey

Abdul Wahab, Nanyang Technological University, Singapore

Hsien-chang Wang, Taiwan, R.O.C.

Jhing-fa Wang, Taiwan, R.O.C.

Wayne Ward, University of Colorado at Boulder, USA

Yukiko Yamaguchi, Nagoya University, Japan

Umit H. Yapanel, University of Colorado at Boulder, USA

Yücel Yemez, Koç University, Turkey

Xianxian Zhang, University of Colorado at Boulder, USA

Lifeng Zhang, Kyushu Institute of Technology, Japan 


\section{Preface}

Over the past thirty years, much progress has been made in the field of automatic speech recognition (ASR). Research has progressed from basic recognition tasks involving digit strings in clean environments to more demanding and complex tasks involving large vocabulary continuous speech recognition. Yet, limits exist in the ability of these speech recognition systems to perform in real-world settings. Factors such as environmental noise, changes in acoustic or microphone conditions, variation in speaker and speaking style all significantly impact speech recognition performance for today systems. Yet, while speech recognition algorithm development has progressed, so has the need to transition these working platforms to realworld applications. It is expected that ASR will dominate the humancomputer interface for the next generation in ubiquitous computing and information access. Mobile devices such as PDAs and cellular telephones are rapidly morphing into handheld communicators that provide universal access to information sources on the web, as well as supporting voice, image, and video communications. Voice and information portals on the WWW are rapidly expanding, and the need to provide user access to larger amounts of audio, speech, text, and image information is ever expanding. The vehicle represents one significant emerging domain where information access and integration is rapidly advancing. This textbook is focused on digital signal processing strategies for improving information access, command and control, and communications for in-vehicle environments. It is expected that the next generation of human-to-vehicle interfaces will incorporate speech, video/image, and wireless communication modalities to provide more efficient and safe operations within car environments. It is also expected that vehicles will become "smart" and provide a level of wireless information sharing of resources regarding road, weather, traffic, and other information that drivers may need immediately or request at a later time while driving on the road. It is also important to note that while human interface technology continues to evolve and expand, the demands placed on the vehicle operator must also be kept in mind to minimize task demands and increase safety.

The motivation for this textbook evolved from many high quality papers that were presented at the DSP in Mobile and Vehicular Systems Workshop, Nagoya, Japan, April 2003, with generous support from CIAIR, Nagoya University. From that workshop, a number of presentations were selected to be expanded for this textbook. The format of the textbook is centered about three themes: (i) in-vehicle corpora, (ii) speech recognition/dialog systems with emphasis on car environments, and (iii) DSP for mobile platforms 
involving noise suppression, image/video processing, and alternative communication scenarios that can be employed for in-vehicle applications.

The textbook begins with a discussion of speech corpora and systems for in-vehicle applications. Chapter 1 discusses a multiple level audio/video/data corpus for in-car dialog applications. Chapter 2 presents the CU-Move invehicle corpus, and an overview of the CU-Move in-vehicle system that includes microphone array processing, environmental sniffing, speech features and robust recognition, and route dialog navigation information server. Chapter 3 also focuses on corpus development, with a study on dialog management involving traffic, tourist, and restaurant information. Chapter 4 considers in-vehicle dialog scenario where more than one user is involved in the dialog task. Chapter 5 considers distributed task management for car telematics with emphasis on VoiceXML. Chapter 6 develops an in-vehicle voice interaction systems for driver assistance with experiments on language modeling for streets, hotels, and cities. Chapter 7 concentrates more on high speech error corrective coding for mobile phone applications which are of interest for car information access. Chapter 8 considers a speech enhancement method for noise suppression in the car environment. Chapter 9 seeks to integrate prosodic structure into noisy speech recognition applications. Effective noise reduction strategies for mobile and vehicle applications are considered in Chapter 10, and also in Chapter 11. Chapter 12 considers a small vocabulary speech system for controlling car environments. Chapters 13 and 14 consider transmission and compression schemes respectively for image and video applications which will become more critical for wireless information access within car environments in the near future. Chapter 15 follows up with a work on adaptive techniques for wireless speech transmission in local area networks, an area which will be critical if vehicles are to share information regarding road and weather conditions while on the road. Chapter 16 considers the use of audio-video information processing to help identify a speaker. This will have useful applications for driver identification in high noise conditions for the car. Chapter 17 considers a rather interesting idea of characterizing driving behavior based on biometric information including gas and brake pedal usage in the car. Chapter 18 addresses convolutional noise using blind signal separation for in-car environments. Finally, Chapter 19 develops a novel approach using multiple regression of the log spectra to model the differences between a close talking microphone and far-field microphone for in-vehicle applications.

Collectively, the research advances presented in these chapters offers a unique perspective of the state of the art for in-vehicle systems. The treatment of corpora, dialog system development, environmental noise suppression, hands-free microphone and array processing, integration of audio-video technologies, and wireless communications all point to the rapidly advancing 
field. From these studies, and others in the field from laboratories who were not able to participate in the DSP in Mobile and Vehicular Systems Workshop [http://dspincars.sdsu.edu/] in April 2003, it is clear that the domain of invehicle speech systems and information access is a rapidly advancing field with significant opportunities for advancement.

In closing, we would like to acknowledge the generous support from CIAIR for the DSP in Mobile and Vehicular Systems Workshop, and especially Professor Fumitada Itakura, who's vision and collaborative style in the field of speech processing has served as an example of how to bring together leading researchers in the field to share their ideas and work together for solutions to solve problems for in-vehicle speech and information systems.

Hüseyin Abut,

San Diego State Univ.
John H.L. Hansen,

Univ. Colorado at Boulder
Kazuya Takeda,

NagoyaUniversity 